\title{
house_prices2
}

\author{
November 11, 2021
}

In this PDF file some links won't work. Find the fully featured Jupyter Notebook file on the website of Prof. Jens Flemming at Zwickau University of Applied Sciences. This work is licensed under a Creative Commons Attribution-ShareAlike 4.0 International License.

\section{Predicting house prices (part 2)}

We try to improve prediction of house prices based on Erdogan Seref's German housing dataset from www.immobilienscout24.de published at www.kaggle.com under a Attribution-NonCommercialShareAlike 4.0 International License.

We load preprocessed data and adjust data types.

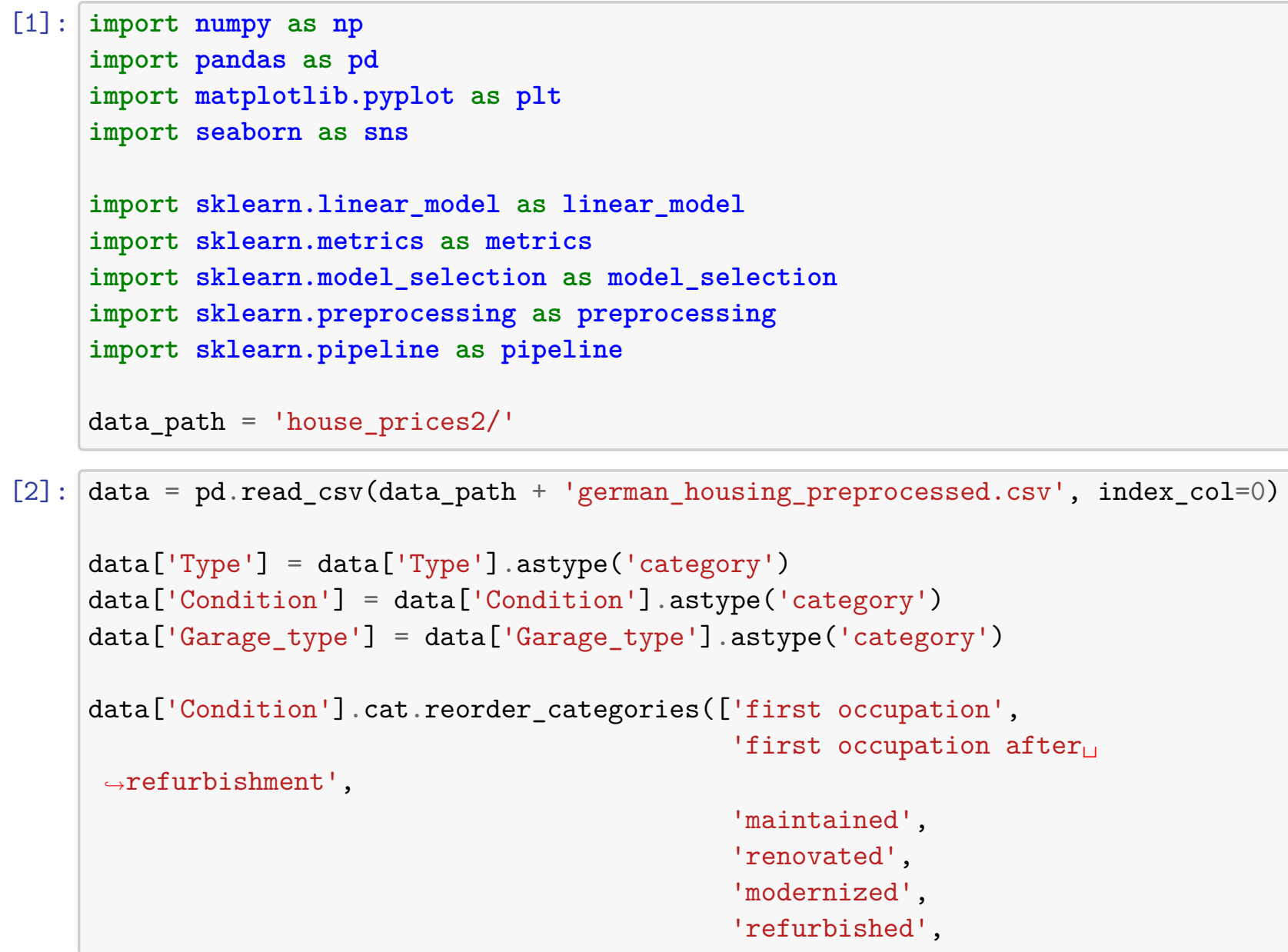


'fixer-upper',

'dilapidated'], inplace=True)

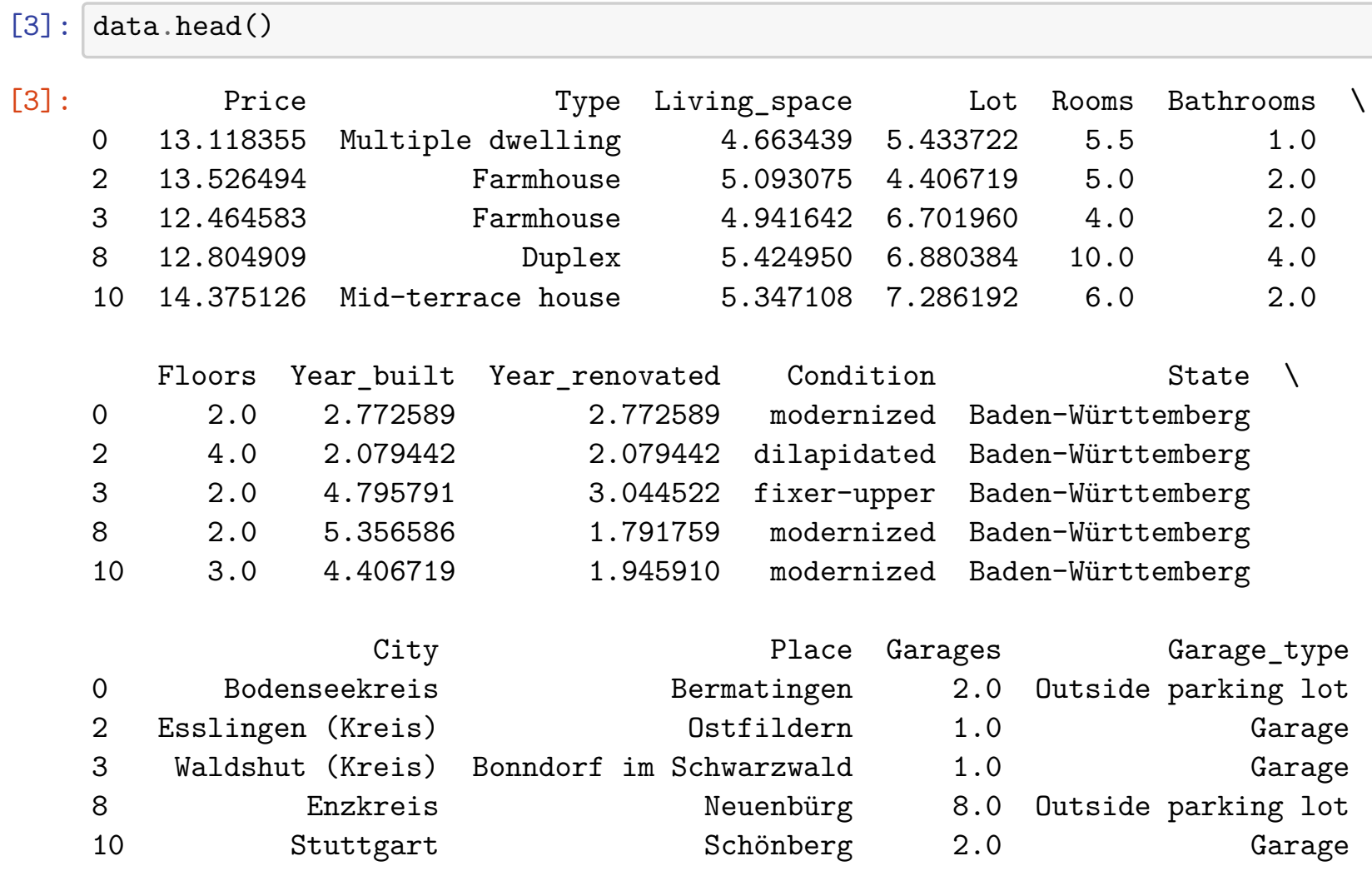

\subsection{More data}

Results obtained from linear regression showed that input variables do not suffice to explain the targets. Thus, we should add more input variables. When preprocessing the data we dropped several columns. Keeping them could increase prediction quality slightly, but there were several good reasons to drop those columns. The main reason were lots of missing values in those columns.

A far better idea is to collect additional data. What features of a house influence the selling price? Of course its location! Up to now we did not use location information at all, but we have location information available. There are columns State, City, Place. But city names do not help. We need something like proximity to big cities or nice landscape. Adding a layer of abstraction we might ask for the demand for houses and the whealth of potential buyers. So we should head out for statistical information about local real estate markets and about economic power of different regions in Germany.

Everything we need is publicly available at www.regionalstatistik.de provided by Statistische Ämter des Bundes und der Länder under the license Datenlizenz Deutschland - Namensnennung - Version 2.0. Clicking here and there we find two interesting tables: * annual income per inhabitant * prices for construction ground

From those tables we may compile a table with 4 columns (region id, region name, income, ground prices). 
The difficult part is matching region names in German housing data set with region names in the region table. Here is some code doing the job:

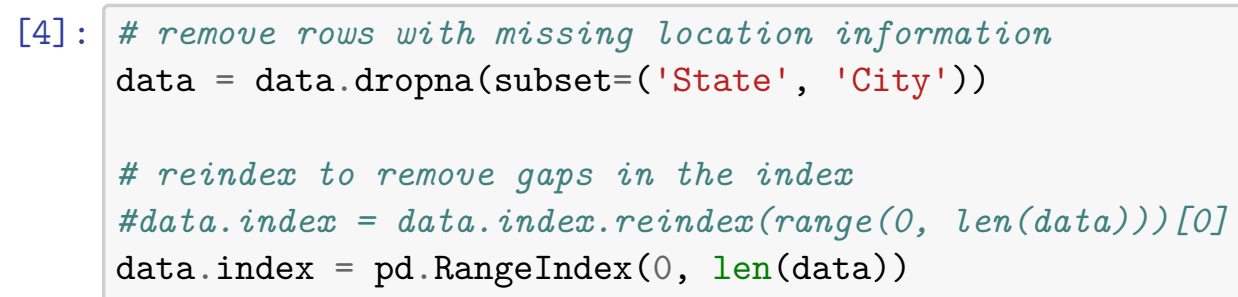

[5]: regions $=$ pd.read_csv(data_path + 'regions.csv')

regions.head (5)

[5]: $\begin{array}{rr} & \text { id } \\ 0 & 0 \\ 1 & 1 \\ 2 & 1001 \\ 3 & 1002 \\ 4 & 1003\end{array}$

$\begin{array}{rrr}\text { region } & \text { income } & \text { prices } \\ \text { Deutschland } & 22623 & 137.67 \\ \text { Schleswig-Holstein } & 22864 & 85.30 \\ \text {, Kreisfreie Stadt } & 19296 & 85.30 \\ \text { t, Kreisfreie Stadt } & 19263 & 85.30 \\ \text { t, Kreisfreie Stadt } & 20363 & 110.84\end{array}$

[6]: data['city_short'] = data['City'].str.replace(' (Kreis)', '', regex=False) data['region_idx'] $=0$

for (idx, city_short) in enumerate(data['city_short']):

find_results = regions['region'].str.find(str(city_short))

if not (find_results $>-1$ ). any ():

\#print (city_short)

if data.loc[idx, 'State'] == 'Hamburg':

find_results $=$ regions['region'].str.find('Hamburg')

data.loc[idx, 'region_idx'] = regions.index[find_results $>-1][-1]$

elif data.loc[idx, 'State'] == 'Bremen':

find_results $=$ regions ['region'].str.find('Bremen')

data.loc[idx, 'region_idx'] = regions.index[find_results $>-1][-1]$

elif data.loc[idx, 'State'] == 'Berlin':

district $=$ city_short.split('(') $[-1][0:-1]$

if district $==$ 'Weißensee':

district $=$ 'Pankow'

if district $==$ 'Prenzlauer Berg':

district $=$ 'Pankow'

if district $==$ 'Hohenschönhausen':

district $=$ 'Lichtenberg'

if district $==$ 'Wedding':

district $=$ 'Berlin-Mitte'

find_results $=$ regions['region'].str.find(district)

\#print ('***', city_short, ':', district, '-->',

$\hookrightarrow$ regions ['Region'] [find_results > -1])

data.loc[idx, 'region_idx'] = regions.index[find_results $>-1][-1]$ 


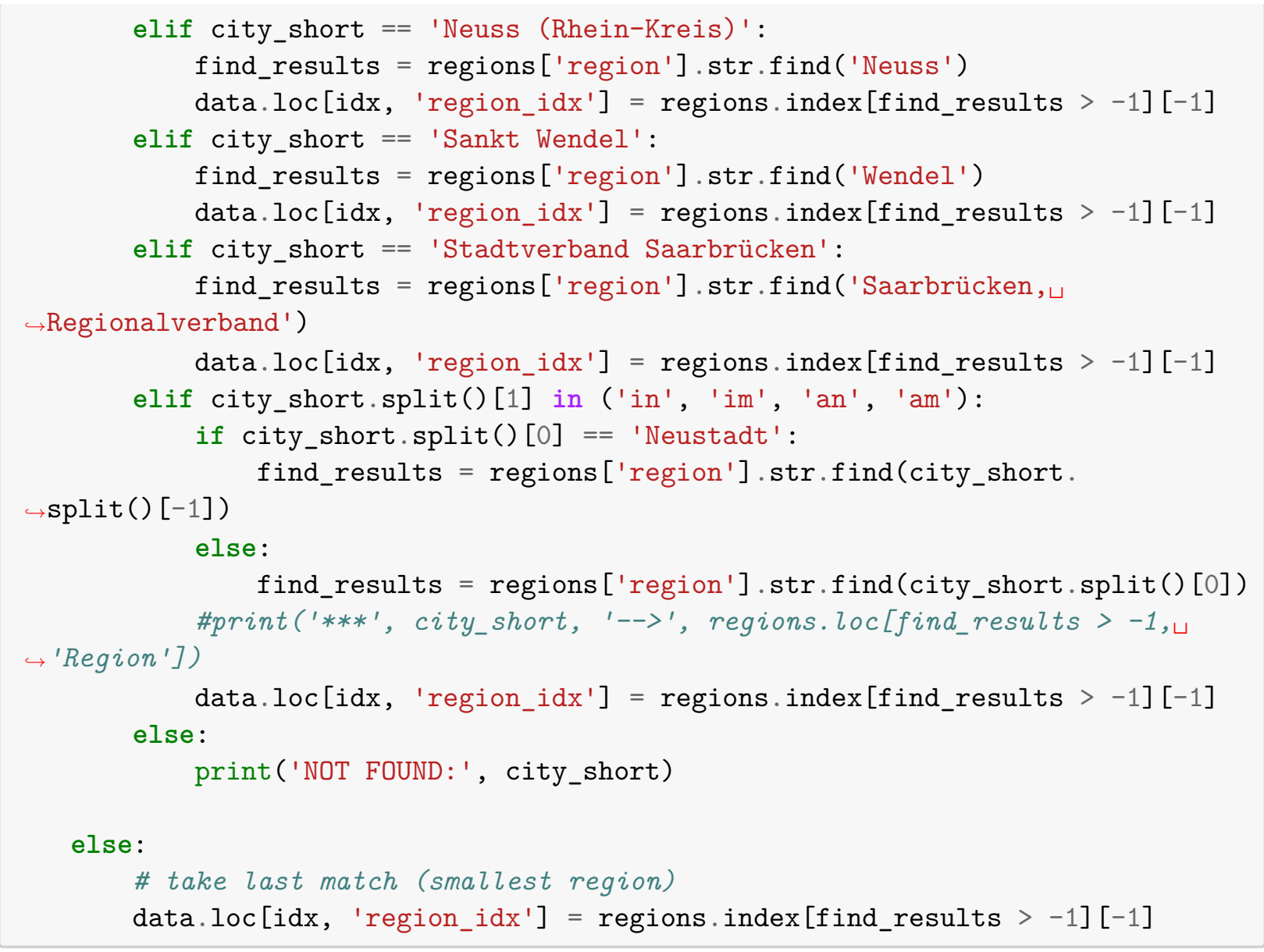

[7]: data['Region_id'] = regions.1oc[data['region_idx'], 'id'].values data['Income'] = regions.loc[data['region_idx'], 'income'].values data['Land_prices'] = regions.loc[data['region_idx'], 'prices'].values

[8]: data $=$ data.drop (columns=['city_short', 'region_idx'] $)$

[9]: data.head (5)

[9] :

$\begin{array}{rrrrrr}\text { Price } & \text { Type } & \text { Living_space } & \text { Lot } & \text { Rooms } & \text { Bathrooms } \\ 13.118355 & \text { Multiple dwelling } & 4.663439 & 5.433722 & 5.5 & 1.0 \\ 13.526494 & \text { Farmhouse } & 5.093075 & 4.406719 & 5.0 & 2.0 \\ 12.464583 & \text { Farmhouse } & 4.941642 & 6.701960 & 4.0 & 2.0 \\ 12.804909 & \text { Duplex } & 5.424950 & 6.880384 & 10.0 & 4.0 \\ 14.375126 & \text { Mid-terrace house } & 5.347108 & 7.286192 & 6.0 & 2.0\end{array}$

$\begin{array}{rrrrr}\text { Floors } & \text { Year_built } & \text { Year_renovated } & \text { Condition } & \begin{array}{r}\text { State } \\ 2.0\end{array} \\ 2.772589 & 2.772589 & \text { modernized } & \text { Baden-Württemberg } \\ 4.0 & 2.079442 & 2.079442 & \text { dilapidated } & \text { Baden-Württemberg } \\ 2.0 & 4.795791 & 3.044522 & \text { fixer-upper } & \text { Baden-Württemberg } \\ 2.0 & 5.356586 & 1.791759 & \text { modernized } & \text { Baden-Württemberg } \\ 3.0 & 4.406719 & 1.945910 & \text { modernized } & \text { Baden-Württemberg }\end{array}$




\begin{tabular}{|c|c|c|c|c|c|c|c|}
\hline \multicolumn{4}{|c|}{ City } & Place & Garages & & Garage_type \\
\hline 0 & \multicolumn{2}{|c|}{ Bodenseekreis } & & Bermatingen & 2.0 & Outside & parking lot \\
\hline 1 & Esslingen & (Kreis) & & Ostfildern & 1.0 & & Garage \\
\hline 2 & Waldshut & (Kreis) & Bonndorf im & Schwarzwald & 1.0 & & Garage \\
\hline 3 & \multicolumn{2}{|c|}{ Enzkreis } & & Neuenbürg & 8.0 & Outside & parking lot \\
\hline 4 & \multicolumn{2}{|c|}{ Stuttgart } & & Schönberg & 2.0 & & Garage \\
\hline & Region_id & Income & Land_prices & & & & \\
\hline 0 & 8435 & 26548 & 172.95 & & & & \\
\hline 1 & 8116 & 25449 & 387.06 & & & & \\
\hline 2 & 8337 & 25304 & 111.64 & & & & \\
\hline 3 & 8236 & 25496 & 192.18 & & & & \\
\hline 4 & 8111 & 25559 & 1500.34 & & & & \\
\hline
\end{tabular}

\subsection{Linear regression}

Now we do linear regression as before, but with two additional columns.

[11]: data['Condition_codes'] = data['Condition'].cat.codes data $=$ pd.get_dummies (data, columns=['Type', 'Garage_type'], drop_first=True)

[12]: $y=\operatorname{data}[$ 'Price'].to_numpy()

$\mathrm{X}=$ data.drop(columns=['Price', 'Condition', 'State', 'City', 'Place', $\hookrightarrow$ 'Region_id']).to_numpy()

print (X.shape, y.shape)

$(4763,23)(4763$,

[13]: X_train, X_test, y_train, y_test = model_selection.train_test_split(X, y, $\hookrightarrow$ test_size $=0.2)$

print(y_train.size, y_test.size)

3810953

[14]: steps $=\left[\left(\right.\right.$ 'poly' $^{\prime}$ preprocessing.PolynomialFeatures ()$)$, ('ridge', linear_model.Ridge())]

pipe $=$ pipeline.Pipeline(steps)

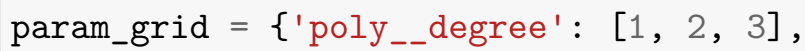
'ridge_alpha': [0] + [2** $\mathrm{k}$ for $\mathrm{k}$ in range $(5,15)]\}$

gs = model_selection.GridSearchCV(pipe, param_grid=param_grid, 


$$
\hookrightarrow \mathrm{CV}=5)
$$

gs.fit(X_train, y_train)

best_params $=$ gs.best_params_

/home/jef19jdw/anaconda3/envs/ds_lecture/lib/python3.7/site-

packages/sklearn/linear_model/_ridge.py:148: LinAlgWarning: Ill-conditioned

matrix (rcond=5.73552e-18): result may not be accurate.

overwrite_a=True). T

\subsection{Evaluation}

Now the interesting part. Do we see an increase in prediction quality?

print (best_params)

pipe.set_params (**best_params)

pipe.fit(X_train, y_train)

y_test_pred $=$ pipe $\cdot$ predict $\left(X_{-}\right.$test $)$

\{'poly__degree': 2, 'ridge__alpha': 512\}

/home/jef19jdw/anaconda3/envs/ds_lecture/lib/python3.7/site-

packages/sklearn/linear_model/_ridge.py:148: LinAlgWarning: Ill-conditioned

matrix ( $r$ cond=5.73552e-18): result may not be accurate.

overwrite_a=True). $\mathrm{T}$

[16] : rmse = np.sqrt (metrics.mean_squared_error (y_test, y_test_pred))

sigma $=n p \cdot s t d\left(y_{-}\right.$test)

print ('RMSE: ', rmse)

print ('standard deviation:', sigma)

print('ratio:', rmse / sigma)

RMSE : 0.4248066771021125

standard deviation: 0.801578549021144

ratio: 0.5299626313863683

[17]: fig, ax = plt. $\operatorname{subplots}()$

ax.plot (y_test, y_test_pred, 'or', markersize=3)

$a x \cdot \operatorname{plot}\left([9,17],[9,17],{ }^{\prime}-b^{\prime}\right)$

ax.set_xlabel ('true targets')

ax.set_ylabel ('predictions')

ax.set_aspect ('equal')

plt.show() 


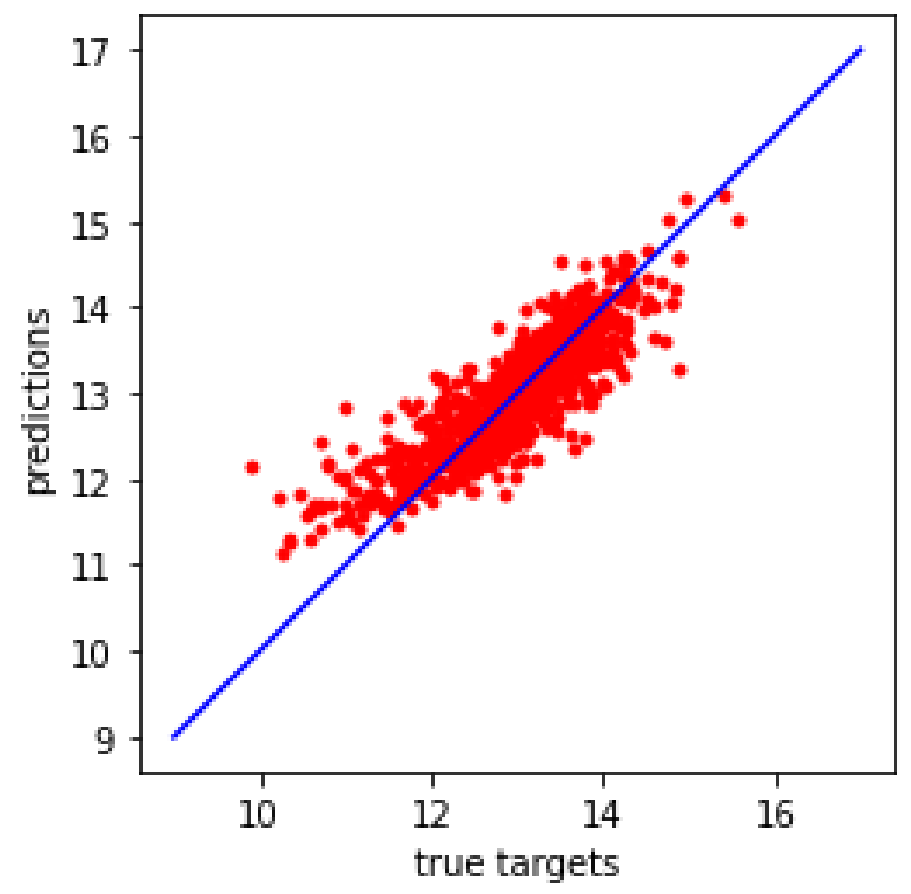

Looks much better!

\subsection{Feature importance}

With a trained model we may look at feature importances to see which features have high influence on the selling price.

[18]: import sklearn.inspection as inspection

[19]: result = inspection.permutation_importance(pipe, $\left.\mathrm{X}, \mathrm{y}, \mathrm{n} \_\mathrm{jobs}=-1\right)$

[20]: cols = data.drop(columns=['Price', 'Condition', 'State', 'City', 'Place', $\hookrightarrow$ 'Region_id']).columns

imp $=$ pd.Series (result.importances_mean, index=cols)

imp = imp.sort_values (ascending $=\mathrm{False}$ )

imp

[20]: Land_prices

Living_space

Year_built

Lot

Income

Rooms

Type_Villa

Bathrooms
0.372617

0.362034

0.174309

0.068924

0.057952

0.031420

0.015153

0.014141 
Type_Duplex

Year_renovated

Garage_type_Outside parking lot

Type_Corner house

Floors

Condition_codes

Type_Mid-terrace house

Garages

Garage_type_Garage

Type_Multiple dwelling

Type_Single dwelling

Type_Farmhouse

Type_Special property

Garage_type_Underground parking lot

Type_Residential property

dtype: float64
0.013529

0.010670

0.009923

0.008855

0.007275

0.007134

0.005969

0.004720

0.003324

0.002968

0.002767

0.001868

0.001690

0.001336

0.000284

[ ] : 